\title{
The Application of Unstructured Grid in Related Numerical
}

Programming

\author{
SHEN Si ${ }^{1, a}$, GUAN Yan-ling ${ }^{2, b}$ \\ ${ }^{1,2}$ school of environmental science and engineering, Chang'an University, Xi'an \\ 710064,Shaanxi,China \\ a405103189@qq.combuanyl1@163.com
}

\begin{abstract}
Key words: unstructured grids; Delaunay; boundary consistency; sliver elements
\end{abstract}
\begin{abstract}
In computational fluid dynamics programming, meshing quality determines the reliability of the results of numerical calculation. For numerical programming in heat and mass transfer of U-type ground heat exchangers (GHE) in ground source heat pump (GSHP) systems, considering the limitation of the structured grid technology for complex geometric model, unstructured grid technology should be adopted. In this paper, combined with the application and research status at home and abroad, the performance characteristics of the commonly used three kinds of unstructured grid technologies are compared, and the applications of three-dimensional Delaunay grid technology in the related fields are introduced, with emphasis on the research status of the two key problems in the application of this technology, namely boundary consistency and sliver elements. The purpose of this paper is to provide help for the numerical calculation programming of U-type GHE in GSHP systems under coupled heat conduction and advection.
\end{abstract}

\section{Introduction}

GSHP technology is a new technology to use the shallow geothermal energy for heating and air conditioning, with its characteristics of energy saving and environmental protection, has developed rapidly in recent years. The core issue of GSHP is the heat transfer performance of GHE, due to the complexity of its heat and mass transfer, usually to make some simplifications such as the soil as a homogeneous, ignore the impact of underground seepage and rock stratum, and decoupling the inside and outside of the U-type pipe in the analytical model and numerical calculation, etc. Especially for simulating the real process of seepage, is beyond the functional category of existing commercial software CFD. To this end, the author put forward the use of programming to solve the problem. However, the meshing of surface geometry is the first question in the numerical programming of GHE.

About meshing, according to the $2 \mathrm{~d}$ problems generally generate triangle or tetrahedron, and generating tetrahedron or hexahedron in $3 \mathrm{~d}$ problems, especially in the most widely used of the triangle and tetrahedron. Based on the topological structure of the grid points, compute grid can be divided into structured grid and unstructured grid. Clearly structured grid has the advantages of simple structure, convenient numerical processing, however, when dealing with complex geometric model, its adaptability has been challenged. Unstructured grid can better adapt to the complex geometric model, facilitate local refinement, which has been widely applied. Research model of GSHP system is divided into U-type pipe, backfill material and soil, U-type pipe is surface, the shape is irregular, it can be thought of meshing with unstructured grid is more reasonable.

\section{Common Methods of Unstructured Grid}

In the late 1960s, the concept of structured grid has been proposed by former Soviet scientist Godunov and American national laboratory Winslow et al. at the same time. Thompson and two other people are proposed using Solution of Elliptic Equations to generate body-fitted grid in 1974, at this point, the researchers have to be more realize the importance of grid generation technique. Due to the 
limitations of structured grid, since the 1980s, unstructured grid has got a rapid development because of its good adaptability to complex geometric model, and has made great progress in recent 30 years. At present, commonly used unstructured grid generation technology mainly include: Delaunay triangulation method (DT), the advancing front method and quadtree/octree method.

\subsection{Delaunay triangulation method (DT)}

In the 1850s, Dirichlet put forward the concept of the Voronoi diagram, DT has developed based on Voronoi diagram, is one of the widely used methods of unstructured grid generation. About 3d case, usually generate tetrahedron grids, the key problems in the process of see below, not repeat them here (see section 3.2.2-3.2.3).

DT has a good mathematical support, good maneuverability, the method is simple, high efficiency, the generated grid cell is the most full. At present, the classic Delaunay triangular subdivision technology has been very mature, but for the convex domain problem cannot guarantee the integrity of solving regional initial grid, in order to solve the problem, researchers make a supplement and improvement to the classical DT, namely constrained DT. Difficulty of applying the algorithm to 3d is boundary recovery and sliver elements, the two key questions are the focus of scholars both at home and abroad in recent years.

\subsection{The advancing front method}

The advancing front method was first put forward by the $L o^{[1]}$ in 1985 , then is improved and extended to the $2 \mathrm{~d}$ adaptive grid generation by Peraire and others ${ }^{[2]}$ in 1987 , then Parikh and Lohner et al. ${ }^{[3,4]}$ successfully extended to $3 \mathrm{~d}$.

Compared with other algorithms, the advancing front method has more flexibility and reliability, can strictly guarantee the integrity of the border, has strong ability of control to the generating unit, but its every step generate only a new unit, and the need for a lot of intersection, contain and distance judgment, need more skills in program implementation.

\subsection{Quadtree/octree method}

In 1983, Yerry and Shephard ${ }^{[5]}$ put forward quadtree/octree method used for meshing for the first time, formed the famous quadtree/octree method. Then many researchers make a improvement of this method, limited quadtree/octree method is proposed, and successfully applied in many fields.

This method can be used for the generation of $2 \mathrm{~d}$ and $3 \mathrm{~d}$ grid of Complex model, and it generates fast, has strong geometrical adaptability, easy to implement density control, but the grid generation is related to the selection of the initial grid and its orientation, the approximation effect of grid boundary is not ideal, boundary mesh quality is poorer, program implementation is very complicated, compared with the above two methods, does not have the advantage.

\subsection{Comparison of three kinds of grid generation method}

To sum up, after comparing the main performance of three grid generation methods summarized as follows ${ }^{[6,7]}$ : in computational efficiency 、 unit density control、 automation degree and regional adaptability, etc., the results of the three methods are almost the same; For the reliability of the program, DT and advancing front method for $2 \mathrm{~d}$ and $3 \mathrm{~d}$ are good, limited quadtree/octree method is good for 2d,but it on mediocre reliability for 3d; For surface mesh generation, three methods all can generate, DT and the advancing front method generating unit quality is better, limited quadtree/octree method is general; For generating unit type, which all can directly generate the triangle and tetrahedron units, the advancing front method and limited quadtree/octree method can directly generate hexahedron after extension, the DT principle can't.

In the grid quality and the complexity of the program, the three methods differ greatly. DT can output 2d grid with better quality, there may be sliver elements in the process of $3 \mathrm{~d}$ mesh generation, but there are solutions, program implementation is relatively easy; The advancing front method to generate $2 \mathrm{~d}$ and $3 \mathrm{~d}$ mesh quality is good, but there is no mature theoretical basis, the most is to rely on experience to solve the problem, need more skills in the program; Limited quadtree/octree grid method is poorer of boundary mesh quality, and the program is very complicated.

From the above comparison shows that compared with other algorithms, DT strictly meet the computational geometry, has good mathematical support, generating grid is of high efficiency and 
characteristic, programming is relatively easy, better reliability. It can be thought of DT is more appropriate for meshing of 3d model of heat exchanger under coupled heat conduction and groundwater advection, based on this, the following article focuses on the related research of the method.

\section{The Three Dimensional DT in the Application of the Programming Calculation}

\subsection{Meshing of ground heat exchanger in the programming}

About the meshing of heat exchanger model based on programming, Fan Rui ${ }^{[8]}$ considering the groundwater flow and complexity of physical model of GHE, vertical U-type pipe equivalent to a single pipe with equivalent diameter based on the interaction of the two pipe legs, the numerical model of GHE under coupled heat conduction and groundwater advection was built. Unstructured grids were generated by using DT and FORTRAN language, but still is to solve the two dimensional problem. Wang Guanjun ${ }^{[9]}$ based on the theory of porous medium and heat seepage coupling, with two square column pipe approximate replace cylindrical pipe, the three-dimensional heat transfer model was established, and using FORTRAN language to write a computer program. Because of the complexity of the unstructured grid, still use structured grid.

\subsection{The three dimensional DT application in related fields}

For 3d unstructured grid partition, because of its professional and complexity, usually as a research subject is widely applied in various fields, such as finite element analysis, aerospace technology, computer graphics, etc.. In recent years, the research focuses on the optimization of grid quality, boundary recovery and sliver elements.

\subsubsection{Basic research to improve the quality of the grid}

Wang Jianhua et al. ${ }^{[10]}$ describe the steps of generating 3d unstructured grid under Delaunay method in detail. Base grid is used to accelerate the generation of tetrahedron mesh. And they use "visibility tests" solve the problem of overlapping of tetrahedron, put forward a algorithm to judging whether point in arbitrary closed area, as shown in Fig. 1 is the result of meshing by this algorithm. Wang Haoli et al. ${ }^{[11]}$ briefly introduces the principle of Delaunay tetrahedral mesh generation, the concept of statistical standard deviation is proposed to judge the unstructured grid quality. It is found that the algorithm is simple and flexible, small amount of calculation and easy to be implemented in practical applications.

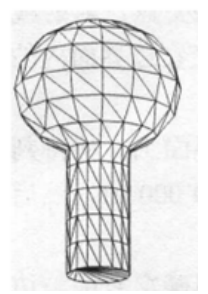

a.The surface grids

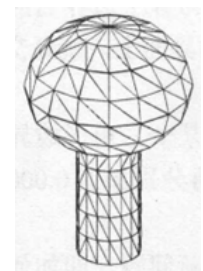

b. The surface grids

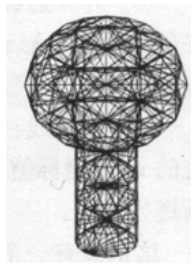

c. Total grids

Fig.1. Round hammer model

\subsubsection{Boundary recovery}

At present, the study of the method for 3d DT is not mature enough, the recovery of boundary edges and boundary surfaces in the designated area is a key problem for study, which should ensure that the output is consistent with the boundary of the model and the initial model. In order to ensure the integrity of the boundary, at present commonly used method is inserted into the Steiner points and edge/surface exchange method, there are also many scholars has carried on the different aspects of the improved variety. Such as the local subdivision, parallel 3d DT algorithm, etc.

(1)In the case of adding auxiliary points

1) The basic algorithm

Chen Jianjun et al. ${ }^{[12]}$ improved the classic 3d Delaunay mesh generation algorithm, add the Steiner point as the auxiliary point, restoring the boundary edges and boundary surfaces by dividing into sections, but the process of boundary recovery involves a large number of intersection 
calculation, the application robustness caused by this is more prominent. Song Chao, Guan Zhenqun, $\mathrm{Gu}$ Yuanxian ${ }^{[13]}$ proposed an effective 3d boundary restoration algorithm of constrained DT subdivision, the algorithm adopts $\alpha$ and $\beta$ operation form pipes in order to restore constrained edges, then according to the number of intersection between constraint surfaces and units type recovery constraint surface to recovery constraint surfaces by types, through the analysis the author found that the method is more complex, hard to master.

2) The improved optimization algorithm

On the basis of the improved constrained Delaunay interpolation point boundary algorithm, Liu $\operatorname{Yan}^{[14]}$ puts forward using arbitrary precision interpolation algorithm to restore the border in failure, the former algorithm is simple, easy to understand, the latter algorithm make the program less dependent on the computer precision, and can reduce the number of the introduction of Steiner points. Based on the Delaunay mesh generation, Wang Shengxi et al. ${ }^{[15]}$ put forward to restore the original model shape by using the Conforming method, and then combined with the Steiner point perturbation technique and vector boundary advancing triangulation method to generate $3 \mathrm{~d}$ constrained tetrahedral mesh, its core is to put $3 \mathrm{~d}$ space constraint problem into a $2 \mathrm{~d}$ grid. A variety of algorithms involved in the restoration process of boundary, need to be used on the basic of relevant theoretical knowledge.

(2)Without adding auxiliary points

Yi Huan ${ }^{[16]}$ studied related research for the boundary recovery of 3d Delaunay mesh generation, the loss of the boundary element leads to the formation of holes in the boundary, finding out all lost surfaces in the boundary holes and all units through the holes to get the shell surfaces of the holes, forming the initial boundary element grid directly to generate the final grids, so as to achieve the purpose of boundary recovery. The algorithm does not introduce auxiliary point, relatively fast. Xiang Chen ${ }^{[17]}$ propose a novel parallel 3d DT algorithm for large-scale simulations on parallel computers. The experimental results demonstrate this method can achieve high parallel performance and perfect scalability, at the same time preserve model boundary feature. But the implementation is based on the abundant knowledge of computers, has a higher threshold to the study of non computer professionals. As shown in Fig.2 is the meshing result of cylindrical model.
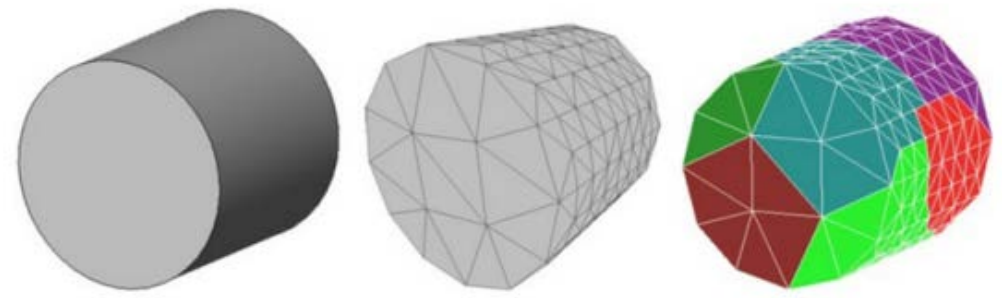
a.Cylinder model
b. Coarse tetrahedral mesh
c. Eight domains after partition

Fig.2.Meshing of cylinder model

\subsubsection{Sliver elements}

Sliver elements appear easily in the process of 3d Delaunay mesh generation. Sliver elements refer to the tetrahedral elements that its four vertices are almost coplanar and Volume is approximate to zero, looking very thin in shape. Because of the bad shape, sliver elements seriously affect the calculation accuracy, even unable to compute, so you must try to eliminate it.

At present, the domestic and foreign scholars have proposed many algorithms to eliminate sliver elements, mainly divided into two major categories of optimization algorithm and encryption algorithm.

(1) Optimization algorithm. The so-called optimization algorithms is to eliminate sliver elements by optimize the generated meshes. Diachin and Smith et al. ${ }^{[18}{ }^{l}{ }^{19}$ put forward to eliminate the sliver elements in the meshes by local transformation of adjacent units with sliver elements. Guan Zhenqun et al. ${ }^{[6]}$ proposed a method called sliver decomposition, by decomposing sliver elements and their adjacent tetrahedral elements, all sliver elements under specified quality factor can be removed. Then on this basis to be improved, a new mesh smoothing method combining the extended sliver 
decomposition method and the Laplacian smoothing method is proposed ${ }^{[20]}$. Using the optimization to eliminate the sliver elements with high efficiency, and the mesh quality is ideal, but optimization effect is poor for sliver elements on the boundary.

(2) Encryption algorithm. The method is by encrypting the mesh to eliminate the sliver elements. Chew and Cheng et al. ${ }^{[21}$ l have different forms by the insertion of new points with encryption method to eliminate the sliver elements. Based on the problems existing in the previous encryption algorithm, Liu Houlin et al. ${ }^{[23]}$ put forward the improvement by combining the minimum dihedral angle with the ratio of the radius of the sphere circumscribing a tetrahedron and the shortest side of tetrahedron in order to determine the sliver elements. Using encryption algorithm to eliminate sliver elements to make full use of the existing grid partition algorithm, and get the reasonable mesh density, the disadvantage is low efficiency.

\section{Conclusion}

This paper briefly introduces the three commonly used methods of unstructured grid, and puts them in comparison. It can be found that DT has a good mathematical support, generating grid is of high efficiency, characteristic, application implementation is relatively easy, for meshing of $3 \mathrm{~d}$ model of heat exchanger under coupled heat conduction and groundwater advection is more suitable. Base on this, this paper mainly expounds the related research and application of 3d Delaunay mesh generation. And it can be found that the main difficulty lies in the problems of boundary recovery and sliver elements. According to the problem of boundary recovery, generally have two methods, one is edge/surface exchange method, the other is the method of inserting the Steiner point. Based on the two methods, researchers also make the improvement or put forward a new idea, and successfully generates a better quality mesh, but stability and complexity remains to be improved. The sliver elements using the corresponding optimization algorithm or encryption algorithm to eliminate, but if you can meet the requirements of efficiency and quality at the same time, the algorithm still needs to be improved.

\section{Acknowledgements}

This work was financially supported by Science And Technology Research Development Program of Shaanxi Province of China (2013K13-02-01) and science and technology plan projects of Shaanxi Province housing and construction department(2014-04).

\section{References}

[1]Lo S H. A new mesh generation scheme for arbitrary planar domains. Int J Numer Methods Eng, 1985,21:1403-1426

[2]Peraire J, Vahdati M, Morgan K, Zienkiewicz O C. Adaptive remeshing for compressible flow. J Comput Phys,1987.72:449-466

[3]Lohner R, Parikh P. Generation of three-dimensional unstructured grids by the advancing front method. Int J Numer Methods Fluids, 1988,8:1135-1149

[4]Parikh P, Pirzadeh S, Lohner R. A package for 3d unstructured grid generation, finite-element flow solution and field visualization. NASA Contract Report 182090,1990

[5] Yerry M A, Shephard M S . A modified quadtree approach to finite element mesh generation[ J] . IEEE Computer Graphics \& Applications , 1983, 3 (1) : 39 -46

[6]Zhenqun Guan,Chao Song, Yuanxian Gu,Xiaofeng Sui. Recent Advances of Research on Finite Element Mesh Generation Methods[J]. JOURNAL OF COMPUTER-AIDED DESIGN \&COMPUTER GRAPHICS,2003 15(1):1-14.

[7]Enqiu Hu,Xinfang Zhang,Wen Xiang,Ji Zhou.A review of mesh generation methods for finite element computation[J]. JOURNAL OF COMPUTER-AIDED DESIGN \&COMPUTER GRAPHICS, 1997,9(4):378-383.

[8]Rui Fan,the oretical and experimental research on GHE in the integrated soil cool thermal storage 
and ground source heat pump system under coupled heat conduction and groundwater advection[D].Harbin Institute of Technology, 2006

[9]Guanjun Wang.Research of Soil Heat Transfer and Groundwater Seepage Based on Elliptic Type Three-dimensional Computation of Convection and Conduction[D]. Chang'an University, Xi'an,2014

[10]Jianhua Wang, Qiangxun Xu, Rui Zhang, Delaunay algorithm and related procedure to generate the tetrahedron mesh for an object with arbitrary boundary[J].Chinese Journal of Rock Mechanics and Engineering, 2003,22(5):717-722

[11]Haoli WANG, Yuan WANG, Binyou YUE,The method and improvement of unstructured tetrahedral mesh generation[J]. J.Xi'an Univ. of Arch.\&Tech.(Natural Science Edition) 2006,38(1):58-62

[12]Jianjun CHEN, Zhengge HUANG, Yongjian YANG , Yao ZHENG , Jianjing ZHENG, Unstructured tetrahedral mesh generation for complex configurations [J],2010,28(4):400-404

[13]Chao Song , Zhenqun Guan, Yuanxian Gu, Boundary restore algorithm and sliver elimination of 3d constrained DT[J]. Chinese Journal of Computational Mechanics, 2004,21(2):169-176

[14]Yan Liu, Robust and efficient constrained Delaunay Tetrahedral mesh generation [D].Dalian University of Technology, Dalian,2010

[15]Shengxi WANG, Songhe SONG, Zhengping ZOU, A 3d Constrained Unstructured Mesh Generation Method with VBATM and Steiner Points Perturbation[J]. CHINESE JOURNAL OF COMPUTATIONAL PHYSICS， 2010,27（5): 649-657

[16]Huan Yi, The method for boundary recovery of 3d Delaunay triangulation method [J]. Jiangsu Aviation, 2011 supplement: 118-121

[17]Xiang Chen, Li Chen, Maode Shi, A highly solid model boundary preserving method for large-scale parallel 3d Delaunay meshing on parallel computers. Computer-Aided Design 58 (2015) 73-83

[18]Diachin L F, Knupp P, Munson T, et al. A comparison of two optimization methods for mesh quality improvement[J]. Engineering with Computers, 2006, 22(2): 61-74.

[19]Smith J M, Dodgson N A. A topologically robust algorithm for Boolean operations on polyhedral shapes using approximate arithmetic[J]. CAD Computer Aided Design, 2007, 39(2): 149-163.

[20]Zhenqun Guan , Bangzhi Liu, Yuanxian Gu , et al. Tetrahedral mesh optimization method combining sliver decomposition and Laplacian smoothing[J]. Chinese Journal of Computational Mechanics, 2007, 24(3): 257-263. (in Chinese with English abstract)

[21]Chew. Guaranteed-quality Delaunay meshing in 3d[C]// Proceedings 13th Annual Symposium on Computational Geometry, Nice France,1997: 391-393.

[22]Cheng W, Poon H. Three-Dimensional Delaunay Mesh Generation[J]. Discrete and Computational Geometry, 2006, 36(3): 419-456.

[23]Houlin Liu , Liang Dong , Minggao Tan,Yong Wang,Kai Wang. Sliver elements elimination for mesh generation of centrifugal pumps [J]. Transactions of the CSAE, 2010,11（26): 103-107 